\title{
Uso da Escala de Satisfação dos Estudantes e Autoconfiança com a Aprendizagem (ESEAA) e da Escala do Design da Simulação (EDS) no ensino de enfermagem: relato de experiência
}

\author{
Use of the Student Satisfaction and Self-Confidence in Learning (SSSCL) and the \\ Simulation Design Scale (SDS) in nursing teaching: experience report
}

\author{
Ellen Cristina Bergamasco ${ }^{1} \bowtie$, Beatriz Murata Murakami ${ }^{1}$, Diná de Almeida Lopes Monteiro da Cruz $^{2}$ \\ ${ }^{1}$ Faculdade Israelita de Ciências da Saúde Albert Einstein. São Paulo, SP, Brasil. \\ 2 Escola de Enfermagem da Universidade de São Paulo. São Paulo, SP, Brasil.
}

Como citar este artigo (How to cite this article):

Bergamasco EC, Murakami BM, da Cruz DALM. Uso da Escala de Satisfação dos Estudantes e Autoconfiança com a Aprendizagem (ESEAA) e da Escala do Design da Simulação (EDS) no ensino de enfermagem: relato de experiência (Use of the Student Satisfaction and SelfConfidence in Learning (SSSCL) and the Simulation Design Scale (SDS) in nursing teaching: experience report). Sci Med. 2018;28(3):ID31036. DOI: 10.15448/1980-6108.2018.3.31036

\section{RESUMO}

OBJETIVOS: Relatar a experiência com o uso da Escala de Satisfação dos Estudantes e Autoconfiança com a Aprendizagem (ESEAA) e da Escala do Design da Simulação (EDS), obtidas a partir da adaptação transcultural da Student Satisfaction and Self-Confidence in Learning (SSSCL) e da Simulation Design Scale (SDS) no curso de Graduação em Enfermagem.

RELATO DA EXPERIÊNCIA: A experiência foi vivenciada em uma instituição de ensino privada da cidade de São Paulo, na disciplina de Saúde do Adulto I, ministrada no $4^{\circ}$ semestre da Graduação em Enfermagem. Essa disciplina conta com dois cenários simulados: atendimento a paciente com alteração glicêmica e atendimento a paciente com desconforto respiratório. Os objetivos da simulação foram discutir raciocínio clínico, conhecimento para identificar o problema principal (diagnóstico de enfermagem) e as habilidades do estudante na realização do cuidado (intervenção de enfermagem). Após a realização das simulações, os alunos foram convidados a preencher os instrumentos ESEAA e EDS CONCLUSÕES: O uso dos instrumentos permitiu identificar o nível de satisfação dos alunos quanto à utilização da simulação como estratégia de ensino e proporcionou aos docentes da disciplina uma reflexão sobre os cenários trabalhados, em termos da clareza do objetivo, conteúdo e condução do debriefing. Os resultados obtidos a partir das respostas dos alunos reforçam a importância de utilização da simulação e estimulam o docente na construção de cenários e no desenvolvimento dessa estratégia.

DESCRITORES: treinamento por simulação; simulação de paciente; educação em enfermagem; inquéritos e questionários.

\section{ABSTRACT}

AIMS: To report the experience with using the Student Satisfaction and Self-Confidence in Learning (SSSCL) and the Simulation Design Scale (SDS) in a Nursing Undergraduate course.

EXPERIENCE REPORT: The experience was held in a private educational institution of the city of São Paulo, in the discipline of Adult Health I, in the 4th semester of Nursing. This discipline has two simulated scenarios: care of a patient with glycemic alteration and care of a patient with respiratory distress. The objectives of the simulation were to discuss clinical reasoning, knowledge to identify the main problem (nursing diagnosis) and the student's abilities to perform care (nursing intervention). After the simulations, the students were invited to fill in the ESEAA and EDS instruments.

CONCLUSIONS: Use of the instruments allowed to identify the level of the students' satisfaction regarding the use of simulation as a teaching strategy and provided the teachers of the discipline with a reflection on the worked scenarios, revising the clarity of the objective, content and conduct of the debriefing. The results obtained from the students' responses reinforce the importance of using simulation and stimulate the teacher in the building of scenarios and developing this strategy.

KEYWORDS: simulation training; patient simulation; education, nursing; surveys and questionnaires. 
Abreviaturas: EDS, Escala do Design da Simulação; ESEAA, Escala de Satisfação dos Estudantes e Autoconfiança com a Aprendizagem; NLN/JSF, National League for Nursing/Jeffries Simulation Framework; SDS, Simulation Design Scale; SSSCL, Student Satisfaction and Self-Confidence in Learning.

\section{INTRODUÇÃO}

A simulação tem sido inserida nos programas de ensino das carreiras da área da saúde, com bons resultados em termos do desenvolvimento de habilidades clínicas, bem como para evitar experiências negativas [1]. Além disso, a Organização Mundial da Saúde recomenda o uso de simulação para garantir a segurança dos pacientes.

Um dos principais interesses no campo de estudos sobre simulação é o desenvolvimento e uso de instrumentos que apoiem os educadores no delineamento, implementação e na avaliação dessa estratégia de ensino $[1,2]$. O modelo teórico para simulação da National League for Nursing (National League for Nursing/Jeffries Simulation Framework$N L N / J S F$ ) [3] tem sido adotado como referência no ensino de enfermagem nos Estados Unidos da América [2] e para os estudos nessa área. O NLN/JSF Simulation Framework tem como pressuposto básico que os resultados da aprendizagem são influenciados pelo professor (facilitador), pelo estudante, pelas práticas educacionais e pelas características da simulação. Como parte do projeto em que o NLN/JSF Simulation Framework foi desenvolvido [3], foram construídos instrumentos de autorrelato para avaliar as percepções e reações dos estudantes à simulação: Simulation Design Scale (SDS), Educational Practices Questionnaire, e Student Satisfaction and Self Confidence in Learning Scale (SSSCL). A SSSCL e SDS foram adaptadas e validadas para a língua portuguesa, sendo, respectivamente, denominados Escala de Satisfação dos Estudantes e Autoconfiança com a Aprendizagem (ESEAA) [4] e Escala do Design da Simulação (EDS) [5]. A ESEAA é constituída por 13 itens, sendo cinco para avaliar a satisfação quanto à atividade de simulação, e oito para avaliar a autoconfiança com a aprendizagem. Para cada item, o respondente deve assinalar em uma escala tipo Likert de cinco pontos o que corresponde à sua percepção: discordo fortemente, discordo, nem concordo nem discordo, concordo ou concordo fortemente. Já a EDS é composta por 20 itens, que avaliam cinco características das simulações desenvolvidas: 1) Objetivos e informação; 2) Apoio; 3) Resolução de problemas; 4) Feedback / Reflexão; e 5) Realismo. Para cada item, o participante deve assinalar duas colunas: uma sobre sua avaliação do item e outra sobre a importância que ele dá para cada item. Na coluna sobre a avaliação do item, em uma escala tipo Likert de cinco pontos, deve assinalar: discordo fortemente, discordo, nem concordo nem discordo, concordo ou concordo fortemente. Na coluna sobre a importância do item, em uma escala tipo Likert de cinco pontos, deve assinalar: não é importante, pouco importante, neutro, importante ou muito importante.

Os instrumentos que obtêm dados de auto percepção e de satisfação dos estudantes podem não refletir resultados de aprendizagem, mas contribuem para o desenvolvimento e aprimoramento da simulação como técnica de ensino [2]. Conhecer a avaliação dos estudantes sobre a estratégia usada, sobre como eles percebem a autoconfiança com a aprendizagem, e sobre a satisfação com a simulação é um retorno para o professor sobre o seu trabalho e lhe fornece elementos para aprimorar a técnica.

Para testar a viabilidade de adotarmos a avaliação das simulações de forma rotineira em um programa de bacharelado em enfermagem, usamos a ESEAA e a EDS em uma sessão de simulação clínica. O objetivo deste artigo é relatar a experiência no uso da ESEAA e da EDS no curso de Graduação em Enfermagem. O relato foi aprovado para publicação pela Direção da Instituição e seguiu os preceitos de ética em pesquisa da Resolução 466/2012 do Conselho Nacional de Saúde, mantendo a confidencialidade da identidade dos participantes.

\section{RELATO DA EXPERIÊNCIA}

A experiência ocorreu em uma instituição de ensino privada na cidade de São Paulo, no curso de Graduação em Enfermagem, que tem carga horária total de 4220 horas distribuídas em oito semestres. As atividades teórico-práticas e o uso da estratégia de simulação clínica permeiam a grade curricular do primeiro ao oitavo semestre. No quarto semestre são ministradas quatro disciplinas: Introdução à Administração em Enfermagem, Geriatria e Gerontologia, Saúde Coletiva I e Saúde do Adulto I - foco em Diagnóstico de Enfermagem, na qual a experiência foi realizada.

A disciplina de Saúde do Adulto I tem carga horária de 135 horas (65 teórico-práticas, 60 de estágio e 10 de educação à distância). Dentre as atividades estão previstas simulações clínicas com objetivo de desenvolver habilidades psicomotoras e comportamentais relacionadas aos conteúdos 
abordados. Para essa disciplina foram construídos dois cenários: atendimento a paciente com alteração glicêmica e atendimento a paciente com desconforto respiratório; ambos foram testados com o intuito de avaliar a pertinência da simulação de acordo com o objetivo da disciplina, nível de dificuldade dos alunos e alinhamento do paciente simulado na condução da situação simulada. Esses cenários foram aplicados por duas vezes nesse mesmo contexto, e no ano de 2016 foram utilizados os instrumentos ESEAA e EDS.

Durante a simulação, os estudantes deveriam identificar a alteração apresentada pelo paciente, nomear o problema principal (diagnóstico de enfermagem) e realizar o cuidado necessário (intervenção de enfermagem). No início da atividade foi solicitado que dois alunos se voluntariassem para a realização da simulação (um aluno para cada cenário).

Os cenários simulados foram realizados no laboratório de habilidades e transmitidos em tempo real para a sala de aula. Cada cenário teve duração de 10 minutos e ao final foi realizado o debriefing com duração de 20 minutos. Após a realização das atividades simuladas, os docentes da disciplina explicaram sobre o uso dos instrumentos, vantagens e desvantagens e os benefícios que poderiam resultar a partir dos resultados; destacou-se que era a primeira vez que a instituição aplicaria a ESEAA e a EDS, e que essa experiência seria relatada posteriormente; os alunos foram convidados a preencher os instrumentos e aqueles que não quiseram preencher foram liberados dessa atividade. Dos 45 alunos participantes da simulação clínica, 38 quiseram participar e responderam aos instrumentos. Os resultados encontrados estão apresentados a seguir.

Em nossa experiência, o escore médio total obtido na ESEAA foi de 4,29 (DP 0,18); na sub escala de Satisfação a média foi de 4,34 (DP 0,17$)$ e na sub escala de Autoconfiança a média foi de 4,24 (DP de $0,19)$. A média poderia variar de 0,0 a 5,0.

Trinta e quatro participantes $(89 \%)$ concordaram ou concordaram fortemente que o método da simulação foi útil e eficaz; 35 (92\%) gostaram do modo como o professor ensinou através da simulação; 31 (82\%) sentiram-se confiantes de que dominavam o conteúdo e $33(87 \%)$ estavam confiantes no desenvolvimento da habilidade a partir da simulação. $O$ resultado de maior destaque da ESEAA foi de que 27 alunos (71\%) referiram que é responsabilidade do professor dizer o que ele precisa aprender na atividade de simulação.

O escore médio obtido pela EDS nessa experiência foi de 4,45 (DP 0,17 ), sendo que $89 \%$ (34) dos alunos referiram que foram fornecidas informações suficientes para os cenários e que esse é um item muito importante no contexto da simulação. Vinte e oito (74\%) alunos referiram que se sentiram apoiados pelo professor durante a simulação e $89 \%$ acreditavam que esse é um item muito importante no contexto da simulação.

Todos os alunos $(100 \%)$ consideraram que a simulação foi projetada para o nível específico de conhecimento e habilidades que eles dispunham no momento; 97\% (37) sinalizaram que a simulação permitiu priorizar avaliações e cuidados de enfermagem e 95\% (36) concordaram que o cenário é semelhante a uma situação real.

\section{DISCUSSÃO}

Em nossa experiência, a escala de satisfação e autoconfiança obteve escores médios muito próximo de 5, mostrando que esses alunos ficaram satisfeitos e confiantes com a experiência de simulação. Sabe-se que a satisfação e autoconfiança do indivíduo com sua aprendizagem são construtos fundamentais dentro do ambiente de ensino [4], daí a importância de avaliá-los durante a prática simulada no ensino de enfermagem.

Dentre os benefícios da satisfação com o aprendizado, afirma-se que a satisfação do aprendiz com a simulação minimiza sentimentos de medo e ansiedade frente à futura profissão [6]. Simulações de alta fidelidade aumentam a satisfação do aluno no aprendizado de habilidades em cuidados intensivos [7] ou em clínica médico-cirúrgica [8]. A melhora da satisfação dos alunos com as atividades de simulação estimula as instituições de ensino a investirem nessa estratégia [9]. A satisfação do aluno favorece o aprendizado, pois exige que o aluno mobilize o conhecimento adquirido e coloque em prática o que foi discutido previamente e permite que o professor exercite e avalie os alunos no que diz respeito à utilização dos conhecimentos, habilidades e competências necessárias para a formação de um bom profissional.

A autoconfiança corresponde à convicção que o indivíduo tem de que é capaz de fazer algo; está relacionada à competência pessoal que a pessoa tem de atingir seus objetivos [10]. Sabe-se que a autoconfiança está diretamente relacionada com competência e sucesso, e pode interferir no desempenho da assistência prestada ao paciente. Enfermeiros autoconfiantes têm melhor desenvolvidas as suas habilidades de análise crítica, reflexão, resolução de problemas e tomada de decisão [11]. Dessa forma, acreditamos que melhorar a autoconfiança dos graduandos em enfermagem pode ajudar na sua formação, possibilitando desenvolver o raciocínio clínico e as habilidades psicomotoras, refletindo assim na melhora do processo ensino-aprendizagem. 
As subescalas da EDS (Objetivos e Informações, Apoio, Resolução de Problemas, Feedback/Reflexão e Realismo) estão relacionadas aos aspectos fundamentais para que a simulação seja planejada com qualidade e sua realização seja feita de maneira efetiva; dessa forma, muitas instituições têm considerado importante validar os cenários e documentar a padronização dos roteiros e objetivos [5].

Uma simulação de qualidade deve ter seu objetivo mensurável, que deve ser específico. O facilitador deve assumir a responsabilidade de guiar a atividade para que o objetivo seja atingido. Os itens da EDS sobre Apoio destacam o papel do instrutor como fundamental na condução da simulação. O debriefing deve acontecer imediatamente após a simulação, pois ajuda a integrar a experiência com sua base de conhecimento [12]. O nível de realismo tem grande influência no processo do aprendizado e contribui para que o participante perceba o quão real está sendo a experiência simulada.

Diante das evidências da literatura e do que vivenciamos em nossa experiência, podemos afirmar que uma atividade simulada de qualidade favorece o bom aproveitamento pelo aluno, e para isso é fundamental que a simulação seja feita de forma adequada, seguindo as melhores práticas e favorecendo o processo de ensino aprendizagem. Cenários construídos e vivenciados de forma adequada permitem que o conteúdo seja aprendido de forma prática em situações próximas às reais. Cabe ao instrutor construir os cenários adequados e conduzir a simulação da melhor forma. Ainda sobre os benefícios do uso das escalas, podemos afirmar que os alunos tiveram que refletir sobre seu conhecimento no assunto para poderem avaliar se dominam ou não o conteúdo, ou ainda que se sentem confiantes no desenvolvimento da habilidade a partir da simulação.

No que diz respeito aos benefícios para os docentes, o uso da ESEAA e da EDS permitiu identificar a satisfação dos alunos com os cenários e os pontos de melhoria que a experiência simulada necessitava. Os docentes puderam reavaliar o objetivo do cenário e o nível de dificuldade do que foi proposto. Estimulou também uma reflexão sobre a percepção de alguns alunos de que, mesmo a simulação sendo uma estratégia ativa de ensino aprendizagem, é responsabilidade do professor dizer o que o aluno precisa aprender. Foi possível identificar a importância que tem para os alunos o apoio do professor durante a simulação, permitindo que os docentes repensem sua prática e reafirmem a sua posição como facilitadores do processo de aprender.

Para as instituições de ensino, o uso das escalas pode avaliar a satisfação do aluno com a estratégia e reforçar a importância da simulação ser inserida na grade curricular e nas atividades planejadas para os futuros enfermeiros. Esses resultados estimulam instituição e docente na continuidade da construção de cenários e desenvolvimento de simulações.

\section{NOTAS}

\section{Agradecimentos}

Agradecemos o apoio da Faculdade Israelita de Ciências da Saúde Albert Einstein (FICSAE). Agradecimentos à equipe do Laboratório da FICSAE e à equipe do Centro de Simulação do Hospital Albert Einstein.

Apoio financeiro

Este estudo não recebeu apoio financeiro de fontes externas.

Declaração de conflito de interesses

As autoras declaram não haver conflitos de interesses relevantes ao conteúdo deste relato de experiência.

Contribuições dos autores

Todas as autoras fizeram contribuições substanciais para concepção, ou delineamento, ou aquisição, ou análise ou interpretação de dados; e redação do trabalho ou revisão crítica; e aprovação final da versão para publicação.

Disponibilidade dos dados e responsabilidade pelos resultados

Todas as autoras declaram ter tido total acesso aos dados obtidos e assumem completa responsabilidade pela integridade destes resultados.

\section{REFERÊNCIAS}

1. Unver V, Basak T, Watts P, Gaioso V, Moss ST, Iwigun E, Tosun N. The reliability and validity of three questionnaires: The Student Satisfaction and Self-Confidence in Learning Scale, Simulation Design Scale, and Educational Practices Questionnaire. Contemporary Nurse. 2017;53(1):60-74. https://doi.org/10.1080/10376178.2017.1282319

2. Tosterud R, Petzäll K, Hedelin B, Hall-Lord ML. Psychometric testing of the Norwegian version of the questionnaire, student satisfaction and self-confidence in learning, used in simulation. Nurse Educ Pract. 2014;14(6):704-8. https://doi. org/10.1016/j.nepr.2014.10.004

3. Jeffries PR and Rizzolo MA. Designing and Implementing Models for the Innovative Use of Simulation to Teach Nursing Care of Ill Adults and Children: A National, Multi-Site, Multi-Method Study. National League for Nursing and Laerdal Medical: New York; 2006. 
4. Almeida RGS, Mazzo A, Martins JCA, Baptista RCN, Girão FB, Mendes IAC. Validação para a língua portuguesa da escala Student Satisfactionand Self-Confidence in Learning. Rev Latino-Am Enfermagem. 2015;23(6):1007-13. https:// doi.org/10.1590/0104-1169.0472.2643

5. Almeida RGS, Mazzo A, Martins JCA, Pedersoli CE, Fumincelli L, Mendes IAC. Validação para a língua portuguesa da Simulation Desgin Scale. Texto Contexto Enferm. 2015;24(4):934-40. https://doi.org/10.1590/0104-0707201500004570014

6. Ying LS, Yiwen K, Rabiah, D. Easing student transition to graduate nurse: a simulated professional learning environment (SIMPLE) for final year student nurses. Nurs Educ Today. 2014;34(3):349-55. https://doi.org/10.1016/j.nedt.2013.04.026

7. Henneman E \& Cunningham H. Using clinical simulation to teach patient safety in an acute/ critical care nurse course. Nurse Educator. 2005;30(4):172-7. https://doi.org/10.1097/00006223-200507000-00010

8. Abdo A \& Ravert P. Student Satisfaction with Simulation Experiences. Clin Simul Nurs Educ. 2006;2(1):13-6. https:// doi.org/10.1016/j.ecns.2009.05.009

9. Baptista RCN, Martins JCA, Pereira F, Mazzo A. Satisfação dos estudantes com as experiências clínicas simuladas: validação de escala de avaliação. Rev Latino-Am Enfermagem. 2014;22(5):709-15. https://doi.org/10.1590/01041169.3295 .2471

10. Bandura A. Social foundations of thought and action: a social cognitive theory. Englewood Cliffs, NJ: Prentice-Hall; 1986.

11. Mazzo A, Martins JCA, Jorge BM, Baptista RCN, Almeida RGS, Henriques FMD, et al. Validation of the self-confidence scale of nursing care in urinary retention. Rev Latino-Am Enfermagem. 2015;23(5):814-20. https://doi.org/10.1590/01041169.0256 .2619

12. Cantrell MA. The importance of debriefing in clinical simulations. Clin Simul Nurs. 2008;4(2):19-23. https://doi. org/10.1016/j.ecns.2008.06.006 